\title{
Interpretation of an international terminology standard in the development of a logic-based compositional terminology
}

\author{
Authors \\ Nicholas R. Hardiker, PhD, RN \\ Senior Research Fellow \\ Salford Centre for Nursing, Midwifery and Collaborative Research \\ University of Salford \\ Room C715 Allerton Building \\ Salford, Greater Manchester \\ M6 6PU, UK
}

Tel: +441612957013

Email: n.r.hardiker@salford.ac.uk

\author{
Amy Coenen, PhD, RN, FAAN \\ Associate Professor \\ College of Nursing \\ University of Wisconsin-Milwaukee
}

USA

Key words: Terminology, Standards, Nursing, International Council of Nurses 
Published as: Hardiker NR, Coenen A. Interpretation of an international terminology standard in the development of a logic-based compositional terminology. International Journal of Medical Informatics. 2007; 76S2: S274-S280.

\section{ABSTRACT}

Purpose Version 1 of the International Classification for Nursing Practice (ICNP®) is a logic-based compositional terminology. ISO 18104:20003 Health Informatics Integration of a reference terminology model for nursing is an international standard to support the development, testing and implementation of nursing terminologies. Methods This study examines how ISO 18104:2003 has been interpreted in the development of ICNP $®$ Version 1 by identifying mappings between ICNP ${ }^{\circledR}$ and the ISO standard. Representations of diagnostic and interventional statements within ICNP ${ }^{\circledR}$ are also analyzed according to the requirements mandated by the ISO standard. Results All structural components of ISO 18104:2003 i.e. semantic categories, semantic domains, qualifiers and semantic links are represented either directly or in interpreted form within ICNP ${ }^{\circledR}$. The formal representations within ICNP ${ }^{\circledR}$ of diagnostic and interventional statements meet the requirement of the ISO standard.

Conclusions The findings of this study demonstrate that ICNP® Version 1 conforms to ISO 18104:20003. More importantly perhaps, this study provides practical examples of how components of a terminology standard might be interpreted and it examines how such a standard might be used to support the definition of high-level schemata in developing logic-based compositional terminologies. 
Published as: Hardiker NR, Coenen A. Interpretation of an international terminology standard in the development of a logic-based compositional terminology. International Journal of Medical Informatics. 2007; 76S2: S274-S280.

\section{CONTRIBUTION OF THIS STUDY}

- The international standard, ISO 18104:2003 Health Informatics - Integration of a reference terminology model for nursing provides a framework for evaluating existing nursing terminologies and a template for developing new ones

- The ISO standard provides no explicit conformance criteria

- Previous studies have examined the level of conformance of nursing terminologies to the ISO standard by decomposing or dissecting terms, and mapping these dissections individually to the standard

- Version 1 of the International Classification for Nursing Practice (ICNP®) is an example of a logic-based compositional terminology

- As such a different approach is needed to assess conformance

- This study exploits the logic-based compositional nature of ICNP® Version 1 and compares the terminology’s high-level schema with the ISO standard

- It provides alternative criteria for conformance to the ISO standard:

o the degree to which the high-level schema for ICNP® Version 1 captures structural components of the standard

o adherence to requirements mandated by the standard of the formal representation within ICNP® Version 1 of diagnostic and interventional statements

- It suggests a possible new role for terminology standards in helping to define high-level schemata for other emerging logic-based compositional terminologies

- Finally it provides practical examples of how the ISO standard and other terminology standards might be interpreted. 
Published as: Hardiker NR, Coenen A. Interpretation of an international terminology standard in the development of a logic-based compositional terminology. International Journal of Medical Informatics. 2007; 76S2: S274-S280.

\section{INTRODUCTION}

Over a decade ago, Sittig included the adoption of a common terminology in his list of grand challenges for health informatics [1]. Since that time, great advances have been made in health care terminology development. For example, in 2003 the International Organization for Standardization (ISO) published the first international standard specifically for health terminologies, ISO 18104:2003 Health Informatics Integration of a reference terminology model for nursing [2]. While the focus for ISO 18104:2003 is on nursing, it is influencing standards intended for other domains. Terminology standards are currently under development within ISO to cover other healthcare domains and are applying ISO 18104:2003 as a model.

For the past few years there has been a general acceptance that health terminologies should be scaled-up, not least to support electronic patient records; and that this scaling-up would not be achievable without computer support [3]. As a result, significant efforts have been directed towards the development of logic-based compositional terminologies $[4,5]$. Version 1 of the International Classification for Nursing Practice [6] (ICNP®) is an example of such a terminology. Other similar terminologies for other domains, such as genomics, are also under development.

Prior to the development of ICNP ${ }^{\circledR}$ Version 1, several terminology experts had already recognized the need for a more formal foundation for nursing terminologies [7, 8]. ISO 18104:2003 provided at least part of that foundation by exposing the semantics embedded within nursing diagnostic and interventional terms. Thus, it provides both a framework for evaluating existing nursing terminologies and a template for developing new ones. 
Published as: Hardiker NR, Coenen A. Interpretation of an international terminology standard in the development of a logic-based compositional terminology. International Journal of Medical Informatics. 2007; 76S2: S274-S280.

Other studies have examined the level of conformance of nursing terminologies to the ISO standard by decomposing or dissecting individual terms, and mapping these dissections to the standard [9-12]. This study goes further than these previous studies. It exploits the logic-based compositional nature of ICNP® Version 1 and compares the terminology's high-level schema with the standard. In so doing it suggests a possible new role for terminology standards in helping to define high-level schemata for other emerging logic-based compositional terminologies and provides examples of how this and other terminology standards might be interpreted.

\section{BACKGROUND}

\section{ICNP ${ }^{\circledR}$ Version 1}

ICNP ${ }^{\circledR}$ Version 1, developed by the International Council of Nurses (ICN), was released in 2005. ICNP® Version 1 built upon previously released versions of ICNP ${ }^{\circledR}$ (alpha, beta, and beta 2) and aims to provide a more formal foundation to support further development and maintenance of the terminology. The core of ICNP® Version 1 (i.e. the ICNP® ontology) is represented in the Ontology Web Language (OWL) [13].

An ontology describes the entities in a domain and the relationships between those entities. OWL is a recommendation of the World Wide Web Consortium (W3C) and is rapidly becoming the de facto standard language for representing ontologies. OWL is useful to ICNP® as it permits subsequent automated (i.e. computerized) description logic reasoning to check consistency and to support classification within the ICNP ${ }^{\circledR}$ ontology, thereby relieving ICN of some of the considerable burden of development.

The ICNP® ontology comprises classes and properties. OWL classes (i.e. concrete representations of entities) are interpreted as sets that contain individuals (i.e. 
Published as: Hardiker NR, Coenen A. Interpretation of an international terminology standard in the development of a logic-based compositional terminology. International Journal of Medical Informatics. 2007; 76S2: S274-S280.

objects in a domain of interest) and are organised into a superclass-subclass hierarchy (i.e. a taxonomy). OWL properties are binary relations on individuals (i.e. linking two individuals together). A simplified graphical representation of the 'hasOnset’ property and the associated relationships that hold between individuals in the classes 'Pain' and 'Acute' is shown in Figure 1.

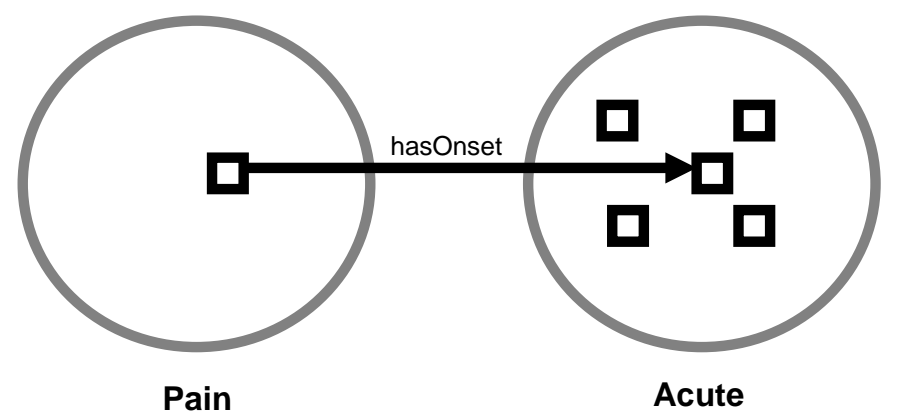

Figure 1: Simplified OWL representation of 'Acute Pain'

\section{ISO 18104:2003}

The stated purpose of the international standard ISO 18104:2003 is "to establish a nursing reference terminology model consistent with the goals and objectives of other specific health terminology models in order to provide a more unified reference health model.” [2]. A reference terminology model is a domain concept model that is optimised for terminology management and a domain concept model is in turn a set of formal categories, semantic links, and sanctions describing potential characteristics for representing concepts in a domain. Thus a nursing reference terminology model may be considered a schema that outlines the basic form of nursing statements.

The standard seeks to support a number of activities:

1. represent nursing concepts (and their relationships) in a manner suitable for computer processing 
2. enable the systematic evaluation of terminologies and associated terminology models for purposes of harmonization

3. facilitate the mapping among nursing diagnosis and nursing action concepts from various terminologies

4. facilitate the construction of nursing terminologies in a regular form i.e. consistency

5. facilitate appropriate integration with other reference terminology models and with information models.

Prior to the development of ICNP® Version 1, ICN co-led the development of ISO 18104:2003 along with the International Medical Informatics Association Special Interest Group on Nursing Informatics. The beta versions of ICNP ${ }^{\circledR}$ contributed greatly to the content of the standard and there are a number of similarities (as shown through the ICNP ${ }^{\circledR}$ 7-axis model in Table 1). However, ICNP ${ }^{\circledR}$ Version 1 is based on a very different underlying technology and the similarities are less obvious.

The standard comprises two models. A graphical representation of the model for statements that describe nursing diagnoses is displayed in Figure 2.

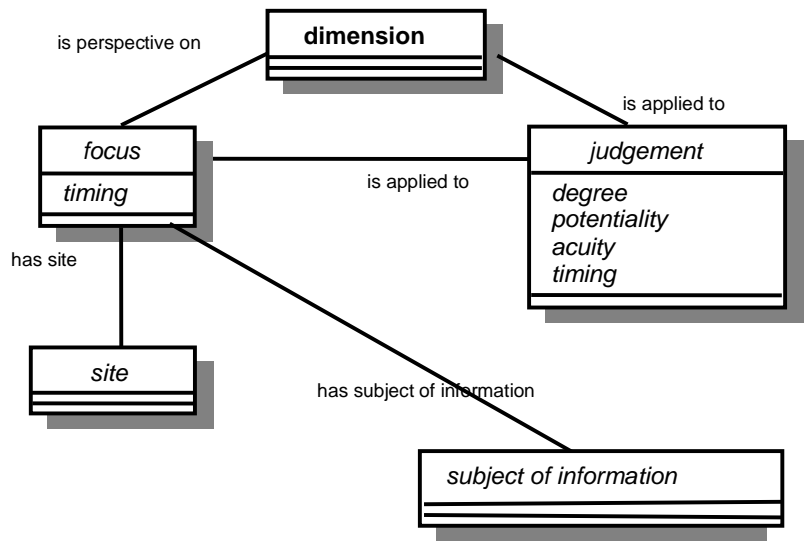

Figure 2: Model for nursing diagnosis 
A graphical representation of the model for statements that describe nursing actions is displayed in Figure 3.

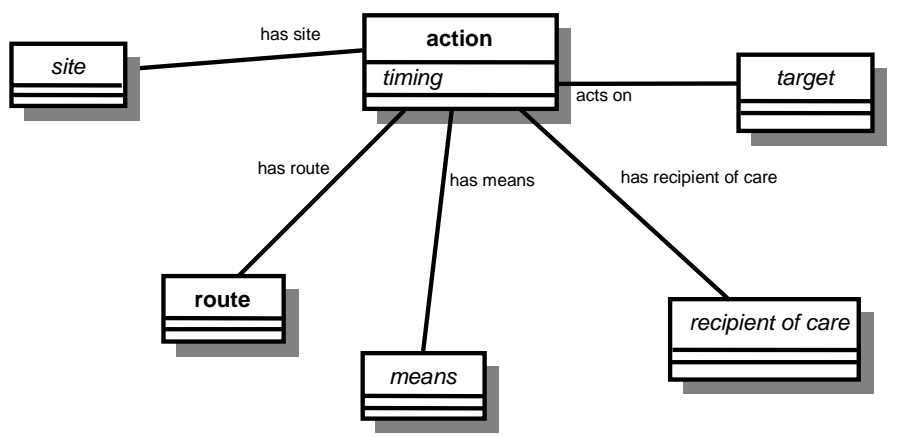

Figure 3: Model for nursing action

Figures 2 and 3 show a number of semantic categories (e.g. dimension) or semantic domains (e.g. focus) - in the upper divisions of the boxes - that are related to one another through various semantic links (e.g. has site) - lines between boxes. Qualifiers - in the central divisions of the boxes - serve to qualify semantic categories and semantic domains.

Semantic categories are shown in bold in both Figures 2 and 3. Semantic categories can be instantiated e.g. an action can exist independently of the model. In contrast, semantic domains, shown in italics, are used to simplify the models and cannot be instantiated e.g. a means cannot exist independently of the model and requires the has means semantic link to make it meaningful.

\section{METHODS}

\section{The axial presentation of ICNP ${ }^{\circledR}$ Version 1}

The software tools used to support the development and maintenance of ICNP® Version 1 allow it to be presented in any number of different ways. One of these ways is the ICNP® 7-axis model [14], an adaptation of ICNP® beta 2 [15]. In this axial 
Published as: Hardiker NR, Coenen A. Interpretation of an international terminology standard in the development of a logic-based compositional terminology. International Journal of Medical Informatics. 2007; 76S2: S274-S280.

presentation, ICNP® concepts are contained within one of 7 axes: Action, Client,

Focus, Judgement, Location, Means, and Time. There are obvious parallels between the axial presentation and the models within the ISO standard. An initial analysis is given in Table 1. 
Published as: Hardiker NR, Coenen A. Interpretation of an international terminology standard in the development of a logic-based compositional terminology. International Journal of Medical Informatics. 2007; 76S2: S274-S280.

Table 1: A comparison between ICNP ${ }^{\circledR}$ axes and ISO 18104:2003 models

ICNP® Axis

ISO Model Element(s)

action

Client

recipient of care

subject of information

\section{Focus}

focus

target

dimension

\begin{tabular}{ll}
\hline Judgement & judgement \\
& degree \\
& potentiality \\
& acuity \\
& \\
\hline Location & site \\
& route \\
\hline Means & means \\
\hline Time & timing \\
\hline
\end{tabular}


Published as: Hardiker NR, Coenen A. Interpretation of an international terminology standard in the development of a logic-based compositional terminology. International Journal of Medical Informatics. 2007; 76S2: S274-S280.

\section{Considering OWL classes and OWL properties}

This cursory analysis suggests that the axial presentation of ICNP ${ }^{\circledR}$ Version 1 would appear to conform to the standard. However, the axial presentation is in many respects artificial. The majority of axis labels within the axial presentation are closely related to semantic domains. In keeping with the standard, these axis labels are not included as OWL classes within the ICNP® Version 1 ontology. As the meaning of semantic domains can only be determined through their associated semantic links, any analysis of conformance of ICNP ${ }^{\circledR}$ Version 1 to the standard must consider not just OWL classes but also OWL properties.

In this study both the OWL classes and OWL properties within the ICNP ${ }^{\circledR}$ Version 1 ontology are examined in order to identify mappings to the semantic categories, semantic domains, qualifiers and semantic links within the standard.

\section{RESULTS}

\section{Semantic categories}

As indicated previously, semantic categories can be instantiated. Thus one would expect to find equivalent OWL classes within the ICNP® Version 1 ontology; and indeed this is the case. The equivalent OWL class for the semantic category action is 'Act', with subclasses such as 'Facilitating Act' or 'Teaching Act'. The equivalent OWL class for the semantic category dimension is 'Status', with subclasses such as ‘Ability’ or 'Knowledge'. Finally, the equivalent OWL class for the semantic category route is 'Route', with subclasses such as 'Gastrointestinal Route' or 'Intravenous Route’.

\section{Semantic domains}


Published as: Hardiker NR, Coenen A. Interpretation of an international terminology standard in the development of a logic-based compositional terminology. International Journal of Medical Informatics. 2007; 76S2: S274-S280.

Also indicated previously is the fact that semantic domains cannot be instantiated and require a semantic link (or, in the case of an OWL ontology, an associated property) to make them meaningful. One would not expect to find a direct correspondence between semantic domains and OWL classes; and within ICNP® Version 1 again this is the case i.e. there are no equivalent OWL classes for semantic domains (or for qualifiers - see Qualifiers). ICNP® Version 1 uses a number of approaches to capture the various semantic domains and qualifiers within the standard.

\section{Means, Target and Site}

The semantic domain means is interpreted within ICNP® Version 1 via the OWL property 'hasMeans'. Any OWL class that is related to a particular 'Act' along the 'hasMeans' property falls within the means semantic domain e.g. in the simplified OWL expression 'MonitoringAct hasMeans MonitoringDevice', 'MonitoringDevice' may be considered a means. (Note that throughout this article, OWL syntax has been greatly simplified to improve readability).

Similarly for target and site. The ICNP ${ }^{\circledR}$ Version 1 ontology has two properties 'actsOn' and 'hasLocation' that are used to represent these semantic domains. In the previous example, 'MonitoringDevice' fell within the semantic domain means. In the following simplified OWL expression, 'MonitoringDevice' now falls within the semantic domain target due to its relationship with 'CleaningAct' along the 'actsOn' property: 'CleaningAct actsOn MonitoringDevice’.

\section{Judgement and Focus}

Judgement is partially interpreted within ICNP $®$ Version 1 as the OWL class 'State' (e.g. 'High’). However, this is only a partial interpretation. In practice an instance of 'State' would only be considered to be a judgement if it was also related to 
Published as: Hardiker NR, Coenen A. Interpretation of an international terminology standard in the development of a logic-based compositional terminology. International Journal of Medical Informatics. 2007; 76S2: S274-S280.

an instance of another OWL class along the 'hasState' property or one of its subproperties.

In parallel with judgement, the semantic domain focus is interpreted within ICNP ${ }^{\circledR}$ Version 1 as anything that has a judgement i.e. it is a focus only when it has a relationship with a particular 'State' along the 'hasState' property or one of its subproperties.

Thus in the simplified OWL expression 'FluidVolume hasState Low', 'FluidVolume' would be considered the locus, and 'Low' would be considered the judgement due to their relationship along the 'hasState' property.

\section{Subject of information and Recipient of care}

Within ICNP ${ }^{\circledR}$ Version 1 the OWL property ‘actsOn’ has a sub-property 'hasRecipientOfCare'. Thus in the simplified OWL expression 'ReassuringAct hasRecipientOfCare Patient', 'Patient' is the recipient of care, and the recipient of care is by definition also a target (according to the ISO model).

Within ICNP ${ }^{\circledR}$ Version 1, interpretation of the semantic domain subject of information is perhaps the most problematic. It is interpreted in several ways, with little consistency in the approach taken. For example, subject of information is interpreted variously through the properties 'isPerformedBy' (e.g. Self-mutilation), ‘isStatusAppliedTo’ (e.g. Caregiver stress), and ‘actsOn’ (e.g. Radiation exposure).

\section{Qualifiers}

The qualifiers degree, potentiality and acuity are interpreted in a similar way to judgement, using specific sub-properties of the OWL property 'hasState' (associated with appropriate sub-classes of the OWL class 'State') to differentiate between them:

- 'hasExtentState' for degree 
- 'hasPotentialityState’ for potentiality

- 'hasOnset' for acuity.

The interpretation of the qualifier timing differs from other qualifiers and semantic domains. The semantics of time are captured partially by the sub-properties of 'hasTime' (e.g. 'occursBefore'), and partially by the values associated with that property. The sub-properties of 'hasTime' act as temporal comparators, capturing expressions such as at, after, before, during, and around. These properties may be associated with time points (e.g. 'Noon'), time intervals (e.g. 'Tomorrow’), time periods (e.g. 'Childhood'), events (e.g. 'Death') or episodes (e.g. 'Operation'). This is consistent with the European standard EN 12381:2005 Health informatics - Time standards for healthcare specific problems [16]. Note that conformance of ICNP® Version 1 to EN 12381:2005 is not the purpose of this study.

\section{Semantic links}

A set of mappings between the semantic links within the standard and the corresponding OWL properties within ICNP® Version 1 is provided in Table 2. 
Published as: Hardiker NR, Coenen A. Interpretation of an international terminology standard in the development of a logic-based compositional terminology. International Journal of Medical Informatics. 2007; 76S2: S274-S280.

Table 2: A comparison between ICNP® OWL properties and semantic links within ISO 18104:2003 models

\section{ISO model semantic link $\quad$ ICNP® OWL property}

\begin{tabular}{ll}
\hline has target & actsOn \\
\hline has degree & hasExtentState \\
\hline has site & hasLocation \\
\hline has route & hasLocation \\
\hline has means & hasMeans \\
\hline has acuity & hasOnset \\
\hline has potentiality & hasPotentialityState \\
\hline has recipient of care & hasRecipientOfCare \\
\hline has timing & hasTime \\
\hline is applied to & isStateOf \\
\hline is perspective on & isStatusAppliedTo \\
\hline has subject of information & isPerformedBy \\
& isStatusAppliedTo \\
\hline & actsOn \\
\hline
\end{tabular}


Published as: Hardiker NR, Coenen A. Interpretation of an international terminology standard in the development of a logic-based compositional terminology. International Journal of Medical Informatics. 2007; 76S2: S274-S280.

Previous studies have also considered the representation of and mapping to semantic links for nursing diagnostic statements [10, 11].

Interesting features of these mappings include:

1. 'hasTime' has different 'flavours' in its sub-properties; these act as temporal comparators

2. 'isStateOf' is not used in the ICNP® Version 1. However, 'hasState', its inverse is used. 'hasState' also has different 'flavours' with sub-properties such as 'hasOnset', 'hasExtentState' and 'hasPotentialityState'.

3. 'actsOn' is a super-class to 'hasRecipientOfCare'.

4. 'hasLocation' is used as a general locative OWL property for the semantic links has site and has route.

5. The semantic link 'hasSubjectOfInformation' is problematic with no single corresponding OWL property, as discussed previously in relation to the semantic domain subject of information.

\section{DISCUSSION}

This study examines how key features of ISO 18104:2003 (i.e. semantic categories, semantic domains, qualifiers and semantic links) have been interpreted in the development of ICNP® Version 1. As there are no explicit conformance criteria within the standard, proxies are used to determine whether the high-level structure of ICNP ${ }^{\circledR}$ conforms to the standard. These proxies include the degree to which ICNP® Version 1 captures components of the standard and the representation of diagnostic and interventional statements.

\section{Representing components of the standard}


Published as: Hardiker NR, Coenen A. Interpretation of an international terminology standard in the development of a logic-based compositional terminology. International Journal of Medical Informatics. 2007; 76S2: S274-S280.

The three semantic categories within the standard i.e. action, dimension and route, have been interpreted as OWL classes within ICNP® Version 1 i.e. 'Act', 'Status', and 'Route'. According to the standard, semantic categories can be instantiated. In OWL, classes are instantiated through individuals i.e. objects in the domain in which we are interested.

Semantic domains have been interpreted within ICNP® Version 1 in a number of ways. Common across all approaches is the fact that within ICNP ${ }^{\circledR}$ Version 1, there are no equivalent OWL classes for any of the semantic domains within the standard; as semantic domains cannot be instantiated, it would make no sense for this to be the case. However, there are means within ICNP ${ }^{\circledR}$ Version 1 of determining which semantic domain a particular OWL class might fall into.

For example with respect to the semantic domain Means, there is no single OWL class within ICNP ${ }^{\circledR}$ Version 1 that corresponds to Means, as one might expect. The most unlikely things can be, and often are, used as the means of carrying out a particular action e.g. a sugar cube as a means of delivering a polio vaccine. However, taken out of context, there is nothing inherent in the definition of those things that would make them a means. They are only a means in the context of the particular action i.e. it is the link with an action that make them a means of carrying out that action e.g. a sugar cube is only a means of delivering a polio vaccine in the context of delivering a polio vaccine; in another context it might well be a means of sweetening coffee or the location of an ant. The ICNP ${ }^{\circledR}$ Version 1 ontology embodies this interpretation through the OWL property 'hasMeans' i.e. any OWL class that is related to a particular 'Act' along the 'hasMeans' property falls within the Means semantic domain. Likewise with the semantic domains Target ('actsOn') and Site ('hasLocation') which have also no 
Published as: Hardiker NR, Coenen A. Interpretation of an international terminology standard in the development of a logic-based compositional terminology. International Journal of Medical Informatics. 2007; 76S2: S274-S280.

independent existence outside the context of a particular action or diagnosis i.e. they themselves cannot be instantiated. Qualifiers are interpreted within ICNP® Version 1 in a similar way to semantic domains.

Of all of the semantic domains, Judgement appears to be the most closely related to a semantic category. Within ICNP® Version 1, all sub-classes of 'State' fall within the semantic domain Judgement and Judgement is covered by 'State'. In other words, the semantic category for 'State' would be the only semantic category within the semantic domain Judgement, and this semantic category would appear in no other semantic domain. 'State' effectively instantiates what is perceived to be the only semantic category within the semantic domain Judgement. In order to conform with the standard however, ICNP® Version 1 would only consider a particular state to be a Judgement if it is related to a Focus along the 'hasState' property in line with the ‘definition' of semantic domain.

Three of the qualifiers within the standard are interpreted in a similar way to Judgement i.e. through a relationship with another OWL class along a sub-property of 'hasState'. Thus, according to ICNP ${ }^{\circledR}$ Version 1, qualifiers within the standard have much in common with semantic domains.

More problematic are the semantic domains Recipient of care and Subject of information. For Recipient of care, the problems appear to stem from the fact that there is no modelling style guide for analysing nursing statements into the models. For example, in the nursing action statement 'Reassuring the patient', the patient might be considered a Target e.g. 'ReassuringAct actsOn Patient'. Equally, the patient might be considered a Recipient of care e.g. 'ReassuringAct hasRecipientOfCare Patient'. The standard states that 'A descriptor for $<$ action $>$ and a descriptor for $<<$ target $>>$ are 
Published as: Hardiker NR, Coenen A. Interpretation of an international terminology standard in the development of a logic-based compositional terminology. International Journal of Medical Informatics. 2007; 76S2: S274-S280.

mandatory for the intensional definition of a nursing action'. If the patient is considered to be a Recipient of care then, according to the standard, this analysis would not constitute a valid nursing intervention statement. If on the other hand the patient is considered to be a Target, then for reasons of completeness, the expression would need to also include the fact that the intervention was performed for the patient, which introduces redundancy e.g. 'ReassuringAct (actsOn Patient hasRecipientOfCare Patient)'. The difficulties are compounded by the problems associated with managing shared variables (i.e. in reality both properties would refer to the same patient).

More serious, and perhaps indicative of a lack of clarity in the scope of the standard, is the inconsistent representation of the semantic domain Subject of information. OWL classes that might fall within the semantic domain Subject of information can be identified within ICNP ${ }^{\circledR}$ Version 1 . However, currently this is a largely manual process.

It is interesting that for both Recipient of care and Subject of information, actual clinical statements concern real people, and specific data about these people would be derived largely from an information model. It may be then that the difficulties with interpreting Recipient of care and Subject of information result from a lack of a clarity in what should form part of a terminology model and what should belong to an information model.

This lack of clarity also affects the semantic links has recipient of care and has subject of information. The remaining semantic links are less problematic, although perhaps some discussion is needed to clarify the interpretation of has site and has route. As discussed previously, route is a semantic category within the standard that has an equivalent OWL class within ICNP® Version 1 . Thus if the OWL property 
Published as: Hardiker NR, Coenen A. Interpretation of an international terminology standard in the development of a logic-based compositional terminology. International Journal of Medical Informatics. 2007; 76S2: S274-S280.

'hasLocation' is associated with a 'Route', one can assume that the semantics concern a route. If on the other hand it is associated with something other than a 'Route', then one can assume that semantics concern something other than a route i.e. a site. Thus the delineation between has site and has route is considered unnecessary within ICNP® Version 1 and the OWL property 'hasLocation' adequately interprets has site and has route, ultimately with no loss of meaning.

\section{Representing diagnostic and interventional statements}

As mentioned previously, the standard has no explicit conformance criteria. It does however state that:

A descriptor for $<<$ focus $>>$ and a descriptor for $<<$ judgement $>>$ are mandatory for the intensional definition of a nursing diagnosis [2] and that

A descriptor for $<$ action $>$ and a descriptor for $<<$ target $>>$ are mandatory for the intensional definition of a nursing action [2]. ICNP ${ }^{\circledR}$ Version 1 embodies these requirements in its formal definitions for nursing diagnosis and nursing intervention. A nursing diagnosis (known within ICNP® Version 1 as ‘DiagnosticPhenomenon’) is defined as: ‘Phenomenon hasJudgedState JudgedState'.

This means that a 'DiagnosticPhenomenon' is anything that has a 'JudgedState'. ‘JudgedState' has two sub-classes 'PositiveState' and 'NegativeState'. This complies with the requirement laid down by the standard while tightening the definition of what is meant by a Judgement (in the context of a nursing diagnostic statement). In essence 'JudgedState' attempts to capture the notion of a judgement either as a positive thing or 
Published as: Hardiker NR, Coenen A. Interpretation of an international terminology standard in the development of a logic-based compositional terminology. International Journal of Medical Informatics. 2007; 76S2: S274-S280.

as a negative thing (this helps for example to delineate between notions of 'Risk' and 'Chance').

A nursing action (known as ‘InterventionalPhenomenon’) is defined as: ‘Act actsOn Phenomenon'. This means that an 'InterventionalPhenomenon' is an Act that acts on (i.e. targets) anything. This again complies with the requirements while also permitting acts that have a particular recipient of care with no explicit Target (due to the subsumption relationship between 'actsOn’ and 'hasRecipientOfCare').

\section{CONCLUSION}

This study identifies criteria to assess conformance to ISO 18104:2003. Using these criteria, from the results of this study we conclude that in terms of representing the components of ISO 18104:2003 (namely semantic categories, semantic domains, qualifiers and semantic links), and in terms of representing diagnostic and interventional statements, ICNP® Version 1 would appear to conform fully to the standard.

This study also provides evidence to inform any future review of ISO 18104:2003, such as the need for explicit conformance criteria, the need for style guides to support the analysis of terminologies (i.e. the dissection of individual terms), and the continued need to clarify the boundaries between terminology models and information models.

More generally, this study provides an example of how to interpret the components of terminology standards in order to support the definition and instantiation of high-level schemata for other emerging logic-based compositional terminologies. Thus it provides direction for terminology developers both within nursing and across other domains. 
Published as: Hardiker NR, Coenen A. Interpretation of an international terminology standard in the development of a logic-based compositional terminology. International Journal of Medical Informatics. 2007; 76S2: S274-S280.

Finally, a clearly stated aim of terminology standards is to influence the development of terminologies. This study demonstrates that terminology development has a role to play in the enhancement of terminology standards.

\section{REFERENCES}

1. Sittig DF. Grand challenges in medical informatics. J Am Med Inform Assoc 1994;1:412-3.

2. International Organization for Standardization. International Standard ISO 18104:2003 Health Informatics—-Integration of a Reference Terminology Model for Nursing. Geneva, Switzerland: International Organization for Standardization, 2003.

3. Rector AL. Clinical Terminology: Why Is it so Hard? Method Inform Med $1999 ; 38: 239-52$.

4. Spackman K, Campbell K, Cote R. SNOMED®RT: A Reference Terminology for Health Care. In: Masys D, ed. AMIA 1997 Annual Symposium. Nashville: Hanley \& Belfus, Inc.; 1997:640-4.

5. Rector A, Bechofer S, Goble C, Horrocks I, Nowlan W, Solomon W. The GRAIL concept modelling language for medical terminology. Artif Intell Med. 1997;9:139-71.

6. International Council of Nurses. Countdown to ICNP® Version $1.3^{\text {rd }}$ issue. Available: http://www.icn.ch/ICNPcountdown.htm. Accessed: 4 August 2005.

7. Hardiker N, Rector A. Modeling nursing terminology using the GRAIL representation language. J Am Med Inform Assoc. 1998;5:120-8. 
Published as: Hardiker NR, Coenen A. Interpretation of an international terminology standard in the development of a logic-based compositional terminology. International Journal of Medical Informatics. 2007; 76S2: S274-S280.

8. Henry S, Mead C. Nursing classifications: Necessary but not sufficient for representing "what nurses do" for inclusion in computer-based patient records. J Am Med Inform Assoc. 1997;4:222-232.

9. Moss J, Coenen A, Mills M.E. Evaluation of the draft international standard for a reference terminology model for nursing actions. J Biomed Inform. 2003;36:271-278.

10. Bakken S, Warren JJ, Lundberg C, et al.. An evaluation of the usefulness of two terminology models for integrating nursing diagnosis concepts into SNOMED®Clinical Terms. Int J Med Inform. 2002;68:71-77.

11. Harris M, Kooim H, Rhudy L, Savova G, Chute C. Testing the generalizability of the ISO model for nursing diagnoses. AMIA Annual Symposium Proceedings. 2003:274-8.

12. Hwang, J.I., Cimino, J., Bakken, S. (2003). Integrating Nursing Diagnostic Concepts into the Medical Entities Dictionary Using the ISO Reference Terminology Model for Nursing Diagnoses. J Am Med Inform Assoc. 10;4: 382-388.

13. World Wide Web Consortium. Web Ontology Language. Available: http://www.w3.org/2004/OWL/. Accessed: 4 August 2005.

14. International Council of Nurses. (2005). International Classification for Nursing Practice-Version 1. Geneva, Switzerland: International Council of Nurses.

15. International Council of Nurses. ICNP® Beta 2. Available: http://www.icn.ch/ICNPupdate.htm. Accessed: 4 August 2005. 
Published as: Hardiker NR, Coenen A. Interpretation of an international terminology standard in the development of a logic-based compositional terminology. International Journal of Medical Informatics. 2007; 76S2: S274-S280.

16. European Committee for Standardisation (CEN). EN 12381:2005. Health informatics - Time standards for healthcare specific problems. Brussels, Belgium: European Committee for Standardisation, 2005. 